\title{
QUALIDADE PERCEBIDA E SATISFAÇÃO DE CLIENTES: UM ESTUDO EXPLORATÓRIO SOBRE OS SERVIÇOS PRESTADOS POR UMA AGÊNCIA DE COMUNICAÇÃO
}

\author{
Gabriel Sperandio Milan \\ Doutor em Engenharia de Produção na área de Sistemas de Qualidade \\ pela Universidade Federal do Rio Grande do Sul (UFRGS). \\ Professor do Departamento de Administração da Universidade de Caxias do Sul \\ (UCS). Coordenador dos cursos de pós-graduação (lato sensu) em Administração \\ Estratégica de Serviços e MBA em Gestão Estratégica da Produção (UCS). \\ Rua: Francisco Getúlio Vargas, 1130 - CEP: 95070-560 - Caxias do Sul - RS \\ gsmilan@ucs.br

\section{Juliana Brentano} \\ Especialista em Marketing pela Universidade de Caxias do Sul (UCS). \\ jubrentano@terra.com.br

\section{Deonir De Toni} \\ Doutor em Administração na área de Marketing pela Universidade Federal \\ do Rio Grande do Sul (UFRGS). Professor do Departamento de \\ Administração da Universidade de Caxias do Sul (UCS). \\ Alameda João Dal Sasso, 800 - CEP: 95700-000 - Bento Gonçalves - RS \\ deonirdt@terra.com.br
}

\section{Resumo}

O presente trabalho apresenta uma pesquisa exploratória realizada junto a uma agência de comunicação de pequeno porte. O estudo está voltado à ampliação do entendimento sobre a satisfação de clientes em relação aos serviços prestados. Neste sentido, por meio de entrevistas individuais, foi realizado um levantamento da 
percepção dos clientes no intuito de verificar quais são os principais atributos do serviço em relação à sua satisfação. Com base em tal análise, foram identificadas, também, as principais oportunidades que a empresa possui para a qualificação dos seus serviços, desdobrando-as em possíveis ações.

Palavras-chave: Qualidade em Serviços, Satisfação de Clientes, Retenção de Clientes.

\begin{abstract}
The current work presents an exploratory research accomplished along with a Communication Agency of small size. The research is directed to the enlargement of the understanding about the customer satisfaction according to the services given. In this matter, through individual interviews, it was accomplished a research of the clients' perception with the objective of checking which the main attributes of the services are according to their satisfaction. With the basis in such analyses, it was identified, also, the main opportunities the business has due to the qualification of its services, unrolling them into possible actions.
\end{abstract}

Key words: Customer Satisfaction, Service Quality, Customer Retention.

\title{
1. Introdução
}

Pesquisar sobre a satisfação dos clientes é uma tarefa fundamental para a gestão das empresas, uma vez que o seu entendimento pode proporcionar uma avaliação de desempenho sob a perspectiva do cliente, indicando decisões tanto estratégicas quanto operacionais que venham a influenciar no nível de qualidade dos serviços prestados pela organização.

Outros benefícios também são evidenciados por diversos estudos, os quais têm descoberto que níveis mais elevados de satisfação levam a maior retenção e lealdade de clientes; e que, com o aumento da lealdade, a satisfação de clientes pode 
ajudar a assegurar receitas ao longo do tempo, reduzir custos de transações futuras, diminuir a elasticidade de preços e minimizar a probabilidade dos clientes trocarem de fornecedor caso haja uma queda no nível da qualidade (ANDERSON; FORNELL; RUST, 1997; ANDERSON, 1998; ANDERSON; FORNELL, 1999).

Reconhecendo a satisfação de clientes como uma medida de desempenho organizacional (ANDERSON, 1998) ou como um indicador de sucesso dos esforços mercadológicos (BABIN; GRIFFIN, 1998), é oportuno abordar o tema pela necessidade premente das empresas em perenizar seus negócios, transformando os clientes em importante ativo, seja por meio de sua retenção, seja por meio de sua lealdade.

Assim sendo, este artigo procura discutir, brevemente, alguns elementos teóricos que auxiliaram na construção do estudo, além de descrever as etapas mais significativas do projeto de pesquisa desenvolvido e, principalmente, apresentar a análise e interpretação das informações coletadas.

\section{Relação entre a Qualidade em Serviços e a Satisfação de Clientes}

No que se refere à qualidade, apesar de sua popularidade, há pouca convergência em torno de sua definição (HUFF; FORNELL; ANDERSON, 1994). Exemplificando, pode-se citar algumas definições de qualidade, as quais abordam diferentes enfoques. Qualidade é conformação a especificações (CROSBY, 1979), melhoria da conformação a especificações através da redução da incerteza e variabilidade (DEMING, 1986), adequação ao uso (JURAN, 1989), julgamento do consumidor sobre a excelência global ou superioridade de um produto ou serviço (ZEITHAML, 1988).

No entanto, Grönroos (1990) e Fornell (1991) comentam que, nesse contexto, o mais importante é a definição do que seja qualidade sob a ótica do cliente. Assim, qualidade é conformação às especificações do cliente e, dessa forma, deve-se considerar aquilo que o cliente percebe como qualidade.

Ao avaliar o desempenho de um produto ou serviço, o cliente geralmente utiliza-se da análise de seus principais atributos (OLIVER, 1997) e, neste sentido, a mensuração do nível de satisfação dos clientes serve como uma medida para se 
verificar o desempenho global da empresa em relação às expectativas dos clientes (ANDERSON; FORNELL; LEHMAN, 1992).

Outras interpretações ainda são derivadas da análise da satisfação. Enquanto os economistas têm se interessado pela aferição da satisfação de clientes como uma representação da avaliação da qualidade, os psicólogos têm se importado com as expectativas e percepções dos clientes como antecedentes da satisfação (JOHNSON; FORNELL, 1991).

O risco das empresas ao interpretarem a satisfação de seus clientes tomando por base a qualidade, está no fato de que os aspectos atinentes à qualidade são determinados, normalmente, pela percepção dos gerentes e, em muitos casos, tal entendimento pode diferir totalmente da visão dos clientes (ZEITHAML; BERRY; PARASURAMAN, 1988).

Dessa forma, a qualidade deve ser vista como o potencial intrínseco aos atributos do produto ou serviço de prover satisfação. Salienta-se, então, que a qualidade percebida é dependente da função utilidade para o cliente em relação ao nível de qualidade ou desempenho fornecido (ANDERSON; FORNELL, 1994). Por sua vez, a satisfação é um constructo que depende da função valor, a qual pode ser definida como o quociente entre a qualidade percebida e o preço, ou pela relação entre os benefícios recebidos pelo cliente comparativamente aos custos ou sacrifícios incorridos para sua obtenção (ANDERSON; FORNELL; LEHMANN, 1992).

Portanto, pode-se entender a qualidade como a percepção atual do cliente sobre o desempenho de um produto ou serviço, e a satisfação como sendo baseada não somente na experiência atual, mas também em experiências passadas coerentemente com o aspecto cumulativo da definição de satisfação postulada por Anderson, Fornell e Lehmann (1992), Johnson e Fornell (1991), Anderson e Fornell (1994) e Garbarino e Johnson (1999) - e em experiências futuras, antecipadas pelo julgamento do cliente devido às suas experiências acumuladas, além da projeção das suas expectativas (FORNELL, 1995).

Para Oliver (1993) e Hallowell (1996), a satisfação está relacionada a uma transação ou compra específica, tratando-a como um julgamento de avaliação, póscompra, de uma ocasião determinada. Entretanto, com enfoque cumulativo, Anderson, Fornell e Lehmann (1992) e Johnson e Fornell (1991) definem satisfação 
de clientes como uma avaliação global baseada em diversas experiências de compra e consumo ao longo do tempo. Anderson e Fornell (1994) também apregoam que a satisfação é uma avaliação contínua a respeito da habilidade de uma marca em proporcionar os benefícios que o cliente está buscando.

Boulding et al. (1993) defendem que o constructo da satisfação apresenta dois conceitos essenciais: a satisfação específica a uma transação e a satisfação cumulativa. Cabe ressaltar que a visão da satisfação específica a uma transação serve para a avaliação de situações específicas, enquanto que a visão cumulativa é mais útil quando o consumidor possui experiências passadas com determinado serviço.

A partir dessa discussão, Oliver (1997) definiu satisfação como a resposta de plenitude do consumidor, ou seja, um julgamento que um produto ou serviço tem como característica, devido ao fato de ter provido ou estar provendo um nível agradável de plenitude relacionada ao consumo, podendo incluir níveis um pouco abaixo (desconfirmação negativa) ou que superam o nível esperado (desconfirmação positiva).

\section{Ambiência da Pesquisa}

A empresa em estudo é uma Agência de Comunicação de pequeno porte, composta pelas suas três sócias-proprietárias, localizada na cidade de Caxias do Sul, no estado do Rio Grande do Sul. Sua área de atuação abrange as cidades de Caxias do Sul e Farroupilha, e seu portfólio de serviços é compreendido por criação, desenvolvimento e implementação de atividades tais como: (i) materiais gráficos (folders, catálogos, panfletos e displays); (ii) mídia externa (outdoors, frontlights e painéis de estrada); (iii) propagandas direcionadas às mídias impressa (jornal e revista), televisão e rádio; (iv) além de toda a parte de identificação visual de empresas (logotipos, materais de expediente e fachadas) e sites corporativos.

Outro aspecto que merece destaque diz respeito à sua carteira de clientes, que conta com 24 clientes de pequeno e médio porte, de diversos segmentos, como, por exemplo, educação, informática, serviços contábeis e em recursos humanos, farmácia de manipulação e saúde, alimentação, entretenimento, vitivinicultura e malharia, dentre outros. 


\section{Definição do Problema e dos Objetivos da Pesquisa}

A definição do problema é a etapa mais importante da pesquisa, pois somente mediante a identificação clara e precisa do problema se torna possível conduzir o trabalho de forma adequada (MALHOTRA, 2001). Sendo assim, o problema de pesquisa se caracteriza pela identificação da percepção dos clientes em relação aos serviços prestados pela Agência e, por consequiência, pela avaliação da satisfação dos clientes, proporcionando à empresa informações que possam embasar ações direcionadas à melhoria da qualidade dos serviços prestados, bem como voltadas à retenção dos clientes.

Por outro lado, foram definidos os seguintes objetivos para a pesquisa:

a) verificar se os clientes da empresa estão satisfeitos ou não em relação aos serviços prestados;

b) identificar quais são os principais atributos relacionados aos serviços prestados e que apresentam maior impacto sobre a satisfação dos clientes;

c) verificar a propensão dos clientes em manter o relacionamento comercial com a empresa;

d) levantar os motivos pelos quais os clientes pretendem manter o relacionamento comercial com a empresa ou pensam em procurar fornecedores alternativos;

e) verificar quais aspectos dos serviços prestados poderiam ser melhorados para que a qualidade percebida dos mesmos seja ampliada; e

f) propor ações a serem tomadas a partir das informações coletadas pela pesquisa.

\section{Método da Pesquisa}

Skinner, Tagg e Holloway (2000) comentam que as técnicas qualitativas focam a experiência das pessoas e seu respectivo significado em relação a eventos, processos e estruturas, inseridos em cenários sociais. Dessa forma, a pesquisa qualitativa utiliza amostras pequenas e estatisticamente não-representativas, prestando-se a ajudar no refinamento de conceitos, a conhecer reações gerais, a 
aprender a linguagem do cliente ou a explorar novas áreas de oportunidade (HELLEBUSCH, 2000).

O principal desafio em investigações qualitativas está na utilização dos dados e não na coleta dos mesmos (WOLCOTT, 1994), uma vez que, a partir de sua utilização, deveria haver a geração de conhecimento capaz de ampliar o entendimento sobre a realidade pesquisada, voltando-se para a compreensão do que pode ser feito e para que direção os dados podem conduzir (RIBEIRO; NEWMANN, 2003). Assim, para os estudos qualitativos, a base para a generalização é a analítica (YIN, 1994), na qual o pesquisador deve expandir e generalizar teorias, e não estabelecer a frequência e a probabilidade nas quais um fenômeno pode ocorrer em uma determinada população (HYDE, 2000).

Para resultar na identificação das percepções dos clientes em relação aos objetivos propostos, os dados foram obtidos (coletados) por meio de entrevistas individuais, de acordo com pressupostos encontrados na literatura (RIBEIRO; NEWMANN, 2003; RIBEIRO; MILAN, 2004).

Neste sentido, para que a coleta de dados fosse representativa, foram resgatados os critérios de classificação de clientes empregados pela empresa (faturamento anual), bem como o potencial de crescimento. Em relação aos participantes, ou seja, cada pessoa que seria entrevistada, representando cada empresa-cliente selecionada, alguns critérios foram observados no que diz respeito ao seu perfil: (i) estar inserida no processo de negociação e/ou decisão da contratação ou manutenção da Agência; (ii) estar em contato com um número expressivo de colaboradores de sua empresa, os quais podem transmitir suas percepções quanto aos serviços prestados pela Agência; e (iii) exercer função ou cargo que lhe confira liberdade de expressão quanto aos aspectos abordados pela pesquisa.

A caracterização dos entrevistados é apresentada no Quadro 1:

Quadro 1 - Caracterização dos entrevistados

\begin{tabular}{|c|c|c|c|}
\hline Entrevistado & Tempo como Cliente (meses) & Cargo & Segmento da Empresa \\
\hline A & 24 & Diretor & Educação em nível superior \\
\hline B & 9 & Sócio-gerente & Tecnologia para vitivinicultores \\
\hline C & 12 & Psicóloga & Serviços contábeis e em RH \\
\hline
\end{tabular}


No intuito de coletar dados e informações com a maior profundidade possível, as entrevistas foram aplicadas, para cada entrevistado, em duas sessões, momentos estes denominados Sessão 1 e Sessão 2 (vide MILAN; RIBEIRO, 2003). As sessões apresentaram um tempo médio de duração de trinta minutos, e foram gravadas em fitas cassete e imediatamente transcritas para posterior análise e interpretação do conteúdo.

Para um maior detalhamento sobre os objetivos de cada sessão, segue o Quadro 2. Destaca-se que cada encontro levou em consideração um roteiro prédefinido de questões elaborado a partir da análise de contexto da organização e adaptações da literatura (MILAN; RIBEIRO, 2003), conforme consta no Anexo, e que, no início da primeira sessão, foi realizada uma breve explanação sobre os objetivos do estudo e de como este seria desenvolvido, assegurando, a cada participante, total sigilo e anonimato em relação às suas colocações.

Quadro 2 - Número de sessões e objetivos

\begin{tabular}{|c|l|}
\hline Sessão & \multicolumn{1}{|c|}{ Objetivo de Cada Sessão } \\
\hline 1 & $\begin{array}{l}\text { Contextualizar a pesquisa para os entrevistados, levantar e registrar dados de forma a } \\
\text { não inibir suas considerações, as quais servirão para consolidar ou reformular o } \\
\text { Roteiro de Questões a ser aplicado na Sessão } 2 .\end{array}$ \\
\hline 2 & $\begin{array}{l}\text { Consolidar e ampliar a coleta de dados através da aplicação ordenada do Roteiro de } \\
\text { Questões. }\end{array}$ \\
\hline
\end{tabular}

Fonte: Milan e Ribeiro (2003, p. 202).

\section{Análise e Interpretação dos Resultados}

No intuito de obter os subsídios necessários para a análise e interpretação dos dados em relação aos objetivos propostos pelo estudo, foram resgatados alguns excertos das três entrevistas realizadas com clientes, contendo os posicionamentos e as observações mais relevantes. 


\subsection{Satisfação dos Clientes em Relação aos Serviços Prestados}

De acordo com os entrevistados, verifica-se que o nível de satisfação em relação aos serviços prestados pela Agência é positivo, o que pode ser ilustrado pelos seguintes comentários:

(...) toda a equipe está satisfeita, (...) de uma maneira geral o trabalho está sendo muito bem aceito, o trabalho é bem feito, de qualidade, (...) considero o trabalho excelente. Resumindo, estou satisfeito (ENTREVISTADO A).

(...) Estou satisfeito, (...) não tive uma demanda maior, mas até então, o que precisei, tudo ok. (...) posso dizer que por ser uma Agência pequena, a gente vê que tem um envolvimento, um trabalho conjunto, eu acho que é por aí. Em relação à empresa, estou satisfeito (ENTREVISTADO B).

(...) Estou bastante satisfeita. (...) É uma relação de parceria. Já tive outras experiências e as pessoas foram oportunistas, (...) o atendimento é dez, os trabalhos são ótimos, a disponibilidade do pessoal é excelente, estou muito satisfeita (ENTREVISTADO C).

\subsection{Atributos do Serviço com Maior Impacto na Satisfação dos Clientes}

$\mathrm{O}$ atributo que apresentou maior impacto na satisfação dos clientes foi a qualidade do atendimento, a qual pode ser melhor entendida como a disponibilidade, o envolvimento com as necessidades dos clientes e a facilidade no processo de comunicação.

O resultado dos trabalhos, a infra-estrutura (instalações e tecnologia) e a localização da Agência também foram citados. Neste sentido, foram resgatados alguns posicionamentos:

(...) o atendimento é excelente, (...) quanto ao atendimento, acho que está perfeito (ENTREVISTADO A).

Segundo o Entrevistado B, os principais aspectos ou atributos relacionados ao serviço que apresentam maior impacto em sua satisfação, respectivamente, são: o atendimento, quanto à afinidade de idéias, facilidade de comunicação, dedicação e 
paciência; destacando, também, a localização da Agência e os resultados provenientes dos trabalhos realizados.

(...) o que mais me deixa satisfeita é o atendimento, o atendimento é dez, as pessoas estão sempre prontas a ver as dificuldades, (...) estão realmente empenhadas para que a solução do problema aconteça o mais rápido possível, então, é isso que mais me chama a atenção. (...) a Agência tem uma ótima localização e uma excelente infra-estrutura comparativamente com o que vejo por aí (ENTREVISTADO C).

\subsection{Propensão à Manutenção do Relacionamento Comercial e seus Motivos}

Todos os entrevistados se mostraram fortemente inclinados a manter o relacionamento comercial entre as partes, sendo que os principais motivos desta intenção está baseada no tipo de relação existente, ou seja, uma relação de confiança, e que vem trazendo bons resultados. Pode-se verificar uma tendência na direção da retenção de clientes, analisando-se alguns excertos das entrevistas:

(...) a relação com a Agência é extremamente produtiva, (...) de confiança, segurança. (...) pretendo manter até o tempo que der, isso depende do pessoal, os resultados têm que aparecer (ENTREVISTADO A).

(...) Até então a relação é boa, estou satisfeito pelo trabalho realizado. Nosso relacionamento é bom, agradável. (...) Sim, pretendo manter a Agência (...) (ENTREVISTADO B).

(...) É uma relação de parceria. (...) Tranqüila, produtiva, séria e de confiança. (...) Com certeza pretendo mantê-la [a Agência] (ENTREVISTADO C).

Por outro lado, reforçando a propensão em relação à manutenção dos relacionamentos existentes, todos os três entrevistados se posicionaram favoravelmente quanto à indicação da Agência a outras pessoas ou empresas, principalmente pela qualidade dos serviços prestados. Como exemplos disso, segue:

(...) tenho falado, mostrado os trabalhos para outras empresas, dizendo que o trabalho é da Agência e é bem feito, excelente (ENTREVISTADO A). 
(...) Eu não só indicaria, como já indiquei para algumas pessoas dentro da área que trabalhamos (...) (ENTREVISTADO B).

\subsection{Oportunidades de Melhoria nos Serviços e Proposição de Ações}

A partir da percepção dos entrevistados, foram identificadas oportunidades de melhoria nos serviços prestados, que podem ser desdobradas em algumas ações, sendo que o principal aspecto diz respeito à agilidade na entrega dos trabalhos, embora a qualidade do atendimento, de forma geral, tenha sido muito elogiada.

(...) a empresa pode ter uma maior agilidade no trabalho [entrega e forma de atendimento]; fui sempre bem atendido, mas sempre tive que procurar os serviços, não vieram na minha empresa (ENTREVISTADO B).

(...) houve um atraso na entrega de um imantado (...) (ENTREVISTADO C).

Além disso, outros elementos identificados incluem a necessidade de explicações mais aprofundadas sobre os serviços que os clientes poderiam implementar (sugestões / indicações) e de um espaço específico para a realização de reuniões nas instalações da Agência, e os preços praticados.

Neste sentido, são sugeridas as seguintes ações:

a) reduzir o prazo de entrega dos trabalhos mediante um estudo aprofundado no processo de criação, desenvolvimento e execução dos serviços prestados;

b) aprofundar as explicações técnicas e possíveis resultados mercadológicos no que tange aos serviços realizados e possíveis alternativas a serem adotadas a fim de ampliar os resultados desejados pelos clientes;

c) dar maior ênfase aos contatos com os clientes, principalmente por meio de visitas pessoais, com o objetivo de aumentar a interação e consolidar os relacionamentos existentes;

d) verificar a possibilidade de implementação de um espaço reservado, nas instalações da Agência, direcionado à realização de reuniões e/ou atendimentos a clientes; e 
e) pesquisar sobre os preços praticados pelos concorrentes, (re)avaliando o posicionamento e a política de preços adotada pela Agência, caso necessário.

\section{Considerações Finais}

Com base na coleta de dados proveniente da pesquisa, pôde-se verificar uma relação significativa entre a qualidade percebida dos serviços prestados, a satisfação e a retenção de clientes, o que está diretamente relacionado ao desempenho organizacional e ao desenvolvimento e sustentabilidade do negócio ao longo do tempo.

Além da preocupação dos clientes com o nível de qualidade do serviço principal, ou seja, os serviços relacionados à comunicação, como por exemplo, materiais gráficos e propagandas, os benefícios advindos dos serviços implícitos, como, por exemplo, da confiança e da segurança no provedor de serviços, evidentemente agregam valor ao relacionamento, o que deve ser reforçado.

Por outro lado, observou-se que a utilização da técnica de entrevistas individuais caracterizou-se como uma técnica adequada para os fins exploratórios da pesquisa, ampliando o entendimento sobre o tema em um contexto específico. A partir da análise e interpretação das informações coletadas, foi possível aferir a percepção dos clientes em relação à empresa e aos serviços prestados, proporcionando uma fundamentação significativa em relação ao processo de tomada de decisões da empresa no que se refere à qualificação dos serviços, à maximização das oportunidades e à consolidação de sua atuação perante o mercado.

\section{REFERÊNCIAS}

ANDERSON, E.W. Customer satisfaction and word-of-mouth. NQRC (National Quality Research Center): The University of Michigan, July 23, 1998.

ANDERSON, E.W.; FORNELL, C. A customer satisfaction research prospectus. In: Service quality: new directions in theory and practice. RUST, R.T.; OLIVER, R.L. (eds.) Thousand Oaks: Sage Publications, p.241-268, 1994. 
ANDERSON, E.W.; FORNELL, C. The customer satisfaction index as a leading indicator. In: Handbook of services marketing and management. IACOBUCCI, D.; SWARTZ, T. (eds.). New York: Sage Publications, 1999.

ANDERSON, E.W.; FORNELL, C.; LEHMANN, D.R. Perceived quality, customer satisfaction, market share, and profitability. Working Paper, NQRC (National Quality Research Center): The University of Michigan, 1992.

ANDERSON, E.W.; FORNELL, C.; RUST, R.T. Customer satisfaction, productivity, and profitability: differences between goods and services. Marketing Science, v.16, n.2, p.129-145; 1997.

BABIN, B.J.; GRIFFIN, M. The nature of satisfaction: an updated examination and analysis. Journal of Business Research, v.41, n.2, p.127-136, 1998.

BOULDING, W.; STAELIN, R.; KALRA, A.; ZEITHAML, V.A. A dynamic process model of service quality: from expectations to behavioral intentions. Journal of Marketing Research, v.30, n.1, p.7-27, 1993.

CROSBY, P.B. Quality is free: the art of making quality certain. New York: New American Library, 1979.

DEMING, W.E. Out of the crisis. Cambridge: MIT Center for Advanced Engineering Study, 1986.

FORNELL, C. National and corporate customer satisfaction indexes. A presentation at the World Quality Day. Amsterdam: World Trade Center, 1991.

FORNELL, C. Productivity, quality, and customer satisfaction as strategic success indicators at firm and national level. Advances in Strategic Management, v.11A, p.217-229, 1995.

GARBARINO, E.; JOHNSON, M.S. The different roles of satisfaction, trust, and commitment in customer relationships. Journal of Marketing, v.63, n.4, p.70-87, 1999.

GRÖNROOS, C. Service management and marketing: managing the moment of truth in service competition. Lexington: Free Press, Lexington Books, 1990.

HALLOWELL, R. The relationships of customer satisfaction, customer loyalty, and profitability: an empirical study. International Journal of Service Industry Management, v.7, n.4, p.27-42, 1996. 
HELLEBUSCH, S. Don't read research by the numbers. Marketing News, v.34, n.19, p.25, 2000.

HUFF, L.; FORNELL, C.; ANDERSON, E.W. Quality and productivity: contradictory and complementary. Working Paper, NQRC (National Quality Research Center): The University of Michigan, 1994.

HYDE, K.F. Recognizing deductive processes in qualitative research. Qualitative Market Research, v.3, n.2, p.82-89, 2000.

JOHNSON, M.D.; FORNELL, C. A framework for comparing customer satisfaction across individuals and product categories. Journal of Economic Psychology, v.12, n.2, p.267-286, 1991.

JURAN, J.M. Juran on leadership for quality. New York: Free Press, 1989.

MALHOTRA, N.K. Pesquisa de marketing: uma orientação aplicada. 3.ed. Porto Alegre: Bookman, 2001.

MILAN, G.S.; RIBEIRO, J.L.D. A influência do departamento de pós-vendas na retenção de clientes: um estudo qualitativo em uma empresa de medicina de grupo. Gestão \& Produção, v.10, n.2, p.197-216, 2003.

OLIVER, R.L. A conceptual model of service quality and service satisfaction: compatible goals, different concepts. Advances in Services Marketing and Management, v.2, p.65-85, 1993.

OLIVER, R.L. Satisfaction: a behavioral perspective on the consumer. New York: Irwin / McGraw-Hill, 1997.

RIBEIRO, J.L.D.; MILAN, G.S. Planejando e conduzindo entrevistas individuais. In: Entrevistas individuais: teoria e aplicações. RIBEIRO, J.L.D.; MILAN, G.S. (eds.). Porto Alegre: FEENG/UFRGS, 2004.

RIBEIRO, J.L.D.; NEWMANN, C.S.R. Planejando a condução de grupos focados. In: Grupos focados: teoria e aplicações. RIBEIRO, J.L.D. (ed.). Porto Alegre: FEENG/UFRGS, 2003.

SKINNER, D.; TAGG, C.; HOLLOWAY, J. Managers and research: the pros and cons of qualitative approaches. Management Learning, v.31, n.2, p.163-179, 2000.

YIN, R.K. Case study research: design and method. $2^{\text {nd }}$ Edition. Thousand Oaks: Sage Publications, 1994. 
WOLCOOT, H.F. Transforming qualitative data: description, analysis, and interpretation. Thousand Oaks: Sage Publication, 1994.

ZEITHAML, V.A. Consumer perceptions of price, quality and value: a means-end model of synthesis of evidence. Journal of Marketing, v.52, n.3, p.2-22, 1988.

ZEITHAML, V.A.; BERRY, L.L.; PARASURAMAN, A. Communication and control processes in the delivery of service quality. Journal of Marketing, v.52, n.2, p.35-48, 1988. 


\section{ANEXO - Roteiro de Questões Utilizado}

\begin{tabular}{|c|c|c|}
\hline $\begin{array}{l}\text { Tipos de } \\
\text { Questão }\end{array}$ & Questões para a Sessão 1 & Questões para a Sessão 2 \\
\hline Iniciais & $\begin{array}{l}\text { 1. Quais os motivos que o levaram a } \\
\text { contatar uma Agência de } \\
\text { Comunicação? } \\
\text { 2. Você se recorda desde quando sua } \\
\text { empresa negocia com a Agência e } \\
\text { como foi o processo de sua escolha? }\end{array}$ & $\begin{array}{l}\text { 13. Recapitulando, em nosso último } \\
\text { encontro, você disse que os principais } \\
\text { aspectos (atributos) do serviço prestado pela } \\
\text { Agência em relação à sua satisfação são os } \\
\text { seguintes:... Você acrescentaria ou excluiria } \\
\text { algum? }\end{array}$ \\
\hline De transição & $\begin{array}{l}\text { 3. Quais são os principais serviços que } \\
\text { você solicita para a Agência? }\end{array}$ & $\begin{array}{l}\text { 14. Quanto à sua percepção relativa aos } \\
\text { pontos fortes e fracos da Agência, você } \\
\text { identificou os seguintes pontos:... Você } \\
\text { confirma esta percepção ou a alteraria? }\end{array}$ \\
\hline Centrais & $\begin{array}{l}\text { 4. Você está satisfeito com os serviços } \\
\text { prestados pela Agência? } \\
\text { 5. Em quais aspectos a Agência e seus } \\
\text { serviços atendem ou não suas } \\
\text { expectativas? } \\
\text { 6. Quais são os principais aspectos } \\
\text { (atributos) do serviço em relação à sua } \\
\text { satisfação? } \\
\text { 7. Na sua opinião, quais são os } \\
\text { principais pontos fortes e pontos fracos } \\
\text { da Agência? } \\
\text { 8. Você já teve alguma experiência } \\
\text { positiva ou negativa com a Agência? } \\
\text { Você poderia relatar? } \\
\text { 9. Como você entende a relação da } \\
\text { Agência com a sua empresa? Por favor, } \\
\text { comente: } \\
\text { 10. Você indicaria a Agência para } \\
\text { alguém? Por quê? }\end{array}$ & $\begin{array}{l}\text { 15. Você já precisou de ajuda do pessoal da } \\
\text { Agência para resolver alguma falha no } \\
\text { serviço? } \\
\text { 16. Se precisou, poderia relatar a situação e } \\
\text { a forma como fora solucionado? } \\
\text { 17. A solução ou o encaminhamento } \\
\text { preencheu sua expectativa quanto ao tempo } \\
\text { de retorno e seu resultado? } \\
\text { 18. Você disse que sua relação com a } \\
\text { Agência é... Você acrescentaria mais algum } \\
\text { comentário? } \\
\text { 19. Como o seu relacionamento, com a } \\
\text { Agência, pode ser caracterizado? } \\
\text { 20. Você disse que indicaria (ou não) a } \\
\text { Agência a outras empresas. Poderia falar } \\
\text { um pouco mais a respeito dos motivos que } \\
\text { baseiam esta tendência? } \\
\text { 21. Foi(ram) sugerido(s) a(s) seguinte(s) } \\
\text { oportunidade(s) de melhoria para a } \\
\text { qualidade dos serviços prestados pela } \\
\text { Agência:... Há mais alguma sugestão? }\end{array}$ \\
\hline Resumo & $\begin{array}{l}\text { 11. Resumindo, como você avalia a sua } \\
\text { satisfação em relação aos serviços } \\
\text { prestados pela Agência? }\end{array}$ & $\begin{array}{l}\text { 22. Resumindo, você está satisfeito com } \\
\text { a qualidade dos serviços prestados pela } \\
\text { Agência? }\end{array}$ \\
\hline Final & $\begin{array}{l}\text { 12. Finalizando, que sugestão você } \\
\text { daria para que os serviços da Agência } \\
\text { fossem melhorados? Comente: }\end{array}$ & $\begin{array}{l}\text { 23. Você pretende manter a Agência como } \\
\text { seu prestador de serviços? Comente: }\end{array}$ \\
\hline
\end{tabular}

Fonte: Elaborado pelos autores. 